\title{
An EXIT-Chart Aided Design Procedure for Near-Capacity N-Component Parallel Concatenated Codes
}

\author{
H. Chen, R. G. Maunder and L. Hanzo \\ School of ECS, University of Southampton, SO17 1BJ, UK. \\ Email: $\{$ hc07r,rm,lh\}@ecs.soton.ac.uk; http://www-mobile.ecs.soton.ac.uk
}

\begin{abstract}
Shannon's channel capacity specifies the upper bound on the amount of bits per channel use. In this paper, we explicitly demonstrate that twin-component turbo codes suffer from a capacity loss, when the component code rate is less than unity, which is shown by exploiting the so-called area properties of Extrinsic Information Transfer (EXIT) charts. This capacity loss is unavoidable for twin-component turbo codes, when the overall turbo coding rate is less than $1 / 2$, while multiple-component turbo codes are capable of overcoming it by using unity-rate component codes. In order to demonstrate that multiplecomponent turbo codes are capable of exhibiting a better asymptotic performance, the minimum Signal Noise Ratio (SNR) required for the EXIT charts to have open convergence tunnels is used as our metric, which is referred to as 'the open tunnel SNR threshold'. Furthermore, the employment of conventional two-dimensional EXIT charts is extended to facilitate the analysis of $N$-component turbo codes. Our results confirm that multiple-component turbo codes approach the Discrete-input Continuous-output Memoryless Channel's (DCMC) capacity more closely and achieve a lower Bit Error Ratio (BER) than twin-component turbo codes at the same coding rate and the same complexity.
\end{abstract}

\section{INTRODUCTION}

Multiple-Component Turbo Codes (MCTC) are constituted by the parallel concatenation of more than two component codes. The concept of MCTCs was proposed by Divsalar as early as 1995 [1, $2]$, where the author introduced the encoder and decoder structures, analyzed the effects of different interleavers and characterized the achievable Bit Error Ratio (BER) performance. Since then, researchers have proposed further beneficial designs $[3,4]$. They mainly used the traditional metric of BER versus Signal Noise Ratio (SNR) and showed that MCTCs may achieve a better performance than conventional Twin-Component Turbo Codes (TCTC). Hence, MCTCs have been widely employed in applications ranging from Hybrid Automatic Repeat reQuest (HARQ) schemes [5] to cooperative relaying networks [6].

For avoiding obfuscating complications, Binary Phase-Shift Keying (BPSK) modulation is employed. According to the area properties of Extrinsic Information Transfer (EXIT) charts [7, Section VIII], for Berrou's classic $N=2$-component turbo codes the area $A$ under a component decoder's EXIT curve is related to both the Discreteinput Continuous-output Memoryless Channel's (DCMC) capacity $C$ and to the component code's rate $R_{c}$, while the overall turbo coding rate is given by $R=R_{c} / N$. More specifically, the following two expressions hold in the case of BPSK modulation:

$$
\begin{array}{cc}
A=C & \left(\text { if } R_{c}=1\right) ; \\
R_{c} \cdot A=C-L & \left(\text { if } R_{c}<1\right),
\end{array}
$$

where $L$ is a positive constant, which depends on both the SNR and the component codes' parameters, physically representing the capacity loss. On the other hand, an open EXIT chart tunnel emerges if the two EXIT curves do not intersect before reaching the point $(1,1)$ of perfect convergence to a vanishingly low BER at a given SNR. More explicitly, this implies that the code is capable of achieving a low BER at this SNR, as long as the interleavers' length

\footnotetext{
${ }^{1}$ The financial support of the China-UK Scholarship Council, of the EPSRC, UK and of the EU under the auspices of the Optimix Project is gratefully acknowledgement.
}

is sufficiently high. Hence, the effective number of bits transmitted per channel use at the corresponding SNR is approximately equal to the current coding rate $R$. Furthermore, in the case of classic $N=2$-component turbo codes, having an area of $A \geq 0.5$ under the decoder's curve implies the presence of an open tunnel and hence a low BER can be achieved. Therefore, Equation 1 transforms to $C \geq 0.5$, i.e. $C \geq R$ under the condition of $2 R=R_{c}=1$. Alternatively, if the component code's rate obeys $2 R=R_{c}<1$, the inequality of $(C-L) \geq R$ derived from Equation 2 demonstrates that a capacity loss is unavoidable, even if the equality is satisfied.

Since typically a strong code associated with a coding rate below $1 / 2$ is required for transmission over hostile channels, classic TCTCs [8] may not constitute attractive choices due to the abovementioned capacity loss. By contrast, MCTCs constituted by $N$ Unity Rate Code (URC) [9] components are attractive turbo codes having a rate of $\frac{1}{N}$. Although the above-mentioned potential capacity loss may be overcome by MCTCs, it is necessary to confirm whether they are capable of approaching the DCMC capacity more closely than TCTCs at the same coding rate, which has not been resolved in the open literature, except that the existence of an SNR difference between the BER curves of the MCTCs found and the Shannon limit was mentioned by Huettinger and Huber [10].

Motivated by solving this open problem, in this paper, we invoke 'the open tunnel SNR threshold' as our metric of estimating the asymptotic performance of MCTCs. EXIT charts [11] [12] play an important role in analyzing the convergence behaviour of classic TCTCs. We will demonstrate that the MCTC decoder may be viewed as being separated into two logical decoder components, each potentially hosting a group of component decoders, where the extrinsic information of all component decoders of a logical decoder will be combined to generate the overall extrinsic output of this logical decoder. This partitioning of the MCTC decoder into two logical parts is necessary, because otherwise we would need an $N$-dimensional EXIT chart for visualizing the extrinsic information exchange of the $N$ component codes. As a benefit, the EXIT chart may be drawn based on the extrinsic information exchange between the two logical decoder parts and then the corresponding 'open tunnel SNR threshold' may be found for the MCTCs.

The rest of this paper is organized as follows. The system model of MCTCs is presented in Section II, while Section III details the 'the open tunnel SNR threshold' metric and describes two feasible methods of drawing the EXIT chart of MCTCs. Section IV provides detailed discussions on our simulation results, which are benchmarked against classic TCTCs. Section V concludes the paper.

\section{SYSTEM MODEL}

Our system model employs URCs as the component codes of the proposed MCTCs. Figure 1 shows the encoder's structure, where the source information is a and its interleaved copies $\left\{\mathbf{a}_{1}, \mathbf{a}_{2}, \mathbf{a}_{3}, \cdots, \mathbf{a}_{N}\right\}$ are entered into the $N$ encoders. The multiplexer seen at the output of the encoder in Figure 1 assembles the encoded bits $\mathbf{b}$, where the resultant bit stream has $N$ times the original sequence length. Hence, the overall coding rate becomes $1 / N$. 


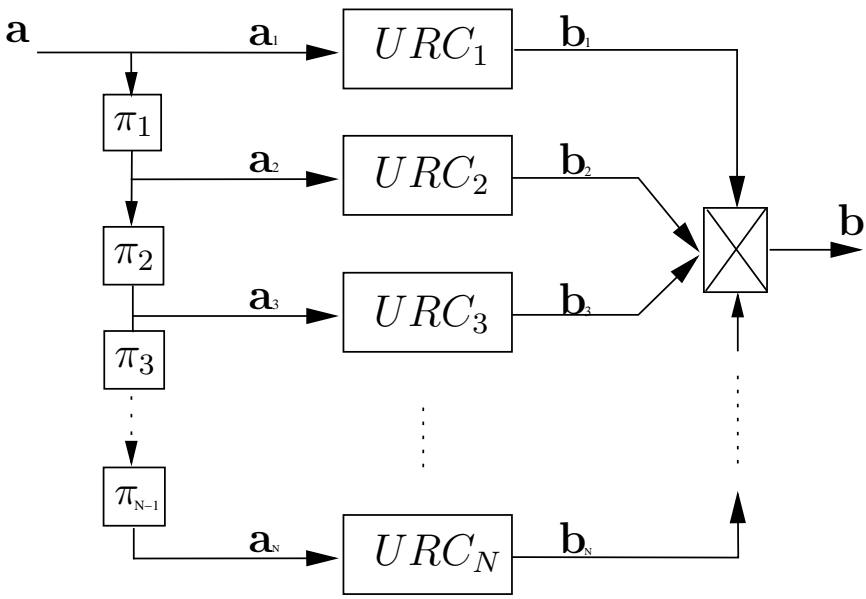

Fig. 1. The encoder structure of a MCTC using $N$ URC components.

Figure 2 shows the corresponding $N$ Bahl-Cocke-Jelinek-Raviv (BCJR) decoders' structure, where each BCJR decoder has two inputs, namely the a priori Log-Likelihood Ratios (LLRs) $\tilde{\mathbf{a}}_{i}^{a}$ combined from all other decoders' extrinsic LLRs and the channel's output information $\tilde{\mathbf{b}}_{i}$. Then each BCJR decoder outputs its own extrinsic LLRs $\tilde{\mathbf{a}_{i}^{e}}$. The decoding process proceeds as follows. Each time a specific BCJR decoder takes control, but numerous schemes have been recommended [12] to choose the decoders' activation order and to appropriately combine the other decoders' extrinsic LLRs. In Section III-A and IV, two specific strategies will be introduced. Decoding operations iterate by exchanging extrinsic information among all $N$ BCJR decoders, until the point of convergence is reached or the affordable number of iterations was exhausted. Then, the recovered bits are decided upon, based on their a posteriori LLRs $\tilde{\mathbf{a}}_{1}^{p}$.

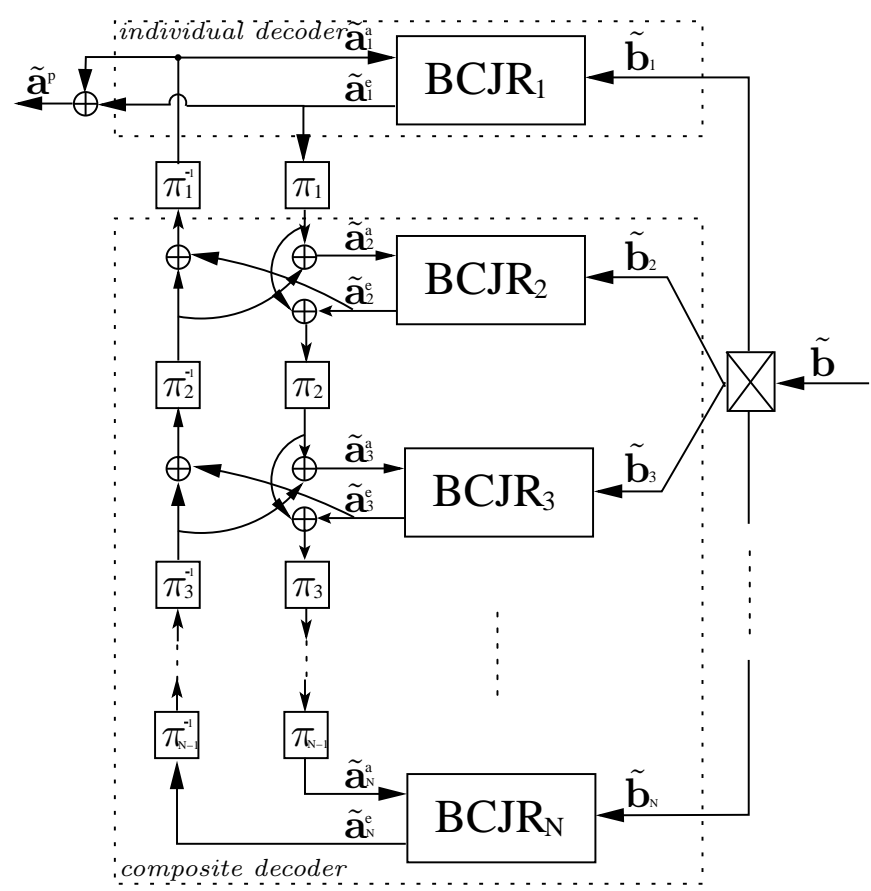

Fig. 2. The decoder structure of a MCTC using $N$ BCJR decoders. Its logical partitioning is showed in the dashed boxes.

\section{EXIT Chart Analysis of Multiple-Component Turbo CODES}

EXIT charts have become a proven technique of analyzing classic TCTCs [12]. In this section, we detail the above-mentioned logical MCTC partitioning method and demonstrate that EXIT chart analysis may also be beneficially applied to design MCTCs. The resultant EXIT charts - which we refer to as extended EXIT charts - directly reflect the convergence behaviour of the MCTCs.

\section{A. Logical partitioning of the MCTC}

For the sake of exploiting the advantages of EXIT charts, we partition the MCTC decoder of Figure 2 into two logical parts, each having a conventional EXIT curve in a conventional EXIT chart. Again, without this partitioning, we would be unable to use 2D EXIT charts to investigate the extrinsic information exchange of $N$ components, because an $N$-dimensional EXIT chart would be required. The two logical parts are surrounded by the dashed rectangles in Figure 2. The MCTC decoder is then treated as the parallel concatenation of an individual URC-BCJR decoder and an $(N-1)$-component composite decoder. As in the EXIT chart analysis of a TCTC, the EXIT function of the individual decoder is expressed as $I\left(\tilde{\mathbf{a}}_{1}^{e}, \mathbf{a}\right)=T_{\text {ind }}\left[I\left(\tilde{\mathbf{a}}_{1}^{a}, \mathbf{a}\right)\right]$, interpreting the a priori Mutual Information (MI) $I\left(\tilde{\mathbf{a}}_{1}^{a}, \mathbf{a}\right)$ as the abscissa and outputting the extrinsic MI $I\left(\tilde{\mathbf{a}}_{1}^{e}, \mathbf{a}\right)$. By contrast, all the $(N-1)$ URC-BCJR decoders seen in the bottom part of Figure 2 are treated as a single amalgamated or composite decoder. The quantity $I\left(\tilde{\mathbf{a}}_{1}^{e}, \mathbf{a}\right)$ output by the upper logical URC-BCJR decoder part of Figure 2 acts as the input a priori $\mathrm{MI}$ of the bottom part, while the combined extrinsic MI of the $(N-1)$-component amalgamated decoder seen in the bottom part of Figure 2 will be passed to the individual stand-alone decoder at the top of Figure 2 as the a priori MI $I\left(\tilde{\mathbf{a}}_{1}^{a}, \mathbf{a}\right)$. The corresponding EXIT function therefore becomes $I\left(\tilde{\mathbf{a}}_{1}^{a}, \mathbf{a}\right)=T_{\text {comp }}$ $\left[I\left(\tilde{\mathbf{a}}_{1}^{e}, \mathbf{a}\right)\right]$. Using this partitioning method, the beneficial attributes of EXIT charts may be essentially retained, despite analyzing $N$ components in two dimensions instead of $N$ dimensions. Hence, the area of the corresponding open EXIT tunnel can be used as per normal for characterizing the achievable performance of MCTCs.

We emphasize here that the a priori LLRs are assumed to obey the Gaussian distribution, but their modeling accuracy can be improved using the experimentally evaluated LLR-histogram. The $(N-1)$ components of the composite decoder iteratively exchange their extrinsic information, until the MI improvements become marginal and hence their convergence is deemed to have been achieved. The resultant combined extrinsic $\mathrm{MI}$ is then fed to the upper individual stand-alone decoder as the current a priori MI.

\section{B. Polynomial-fitting based drawing of EXIT functions}

As briefly alluded to above, instead of assuming that the LLRs are Gaussian distributed, often more accurate results may be generated by experimentally evaluating the LLR-histogram for drawing EXIT charts. However, this method has two potential disadvantages. It is sensitive to the interleaver's design, since it is specific for the particular interleaver used, hence potentially preventing the analysis of the asymptotically attainable performance. Moreover, the task becomes very time consuming, since the length of interleavers should be as high as possible in order to reduce the correlation among the interleaved a priori and extrinsic LLRs. Hence, we propose a semi-analytical polynomial-fitting based method for mitigating the effect of both the interleaver's design and that of its length, which facilitates the creation of the extended EXIT charts of MCTCs. More specifically, each MCTC decoder's EXIT function is modeled using a 
$10^{t h}$-order polynomial generated by curve-fitting, which is expressed as

$$
I\left(\tilde{\mathbf{a}}_{i}^{e}, \mathbf{a}\right)=T_{i n d}^{\prime}\left[I\left(\tilde{\mathbf{a}}_{i}^{a}, \mathbf{a}\right)\right],
$$

where $I\left(\tilde{\mathbf{a}}_{i}^{a}, \mathbf{a}\right)$ is generated on the basis of combining all the other $(N-1)$ URC-BCJR decoders' extrinsic information. Our goal is then to find the appropriate expression for combining each of the $(N-1)$ URC-BCJR decoder's extrinsic information for the sake of generating $I\left(\tilde{\mathbf{a}}_{i}^{a}, \mathbf{a}\right)$ for each individual decoder, one-by-one.

Based on the theory of information combining [13], it is indeed possible to find the expression of $I\left(\tilde{\mathbf{a}}_{i}^{a}, \mathbf{a}\right)$, which is generated from all the other $(N-1)$ sources of extrinsic information given by $I\left(\tilde{\mathbf{a}}_{j}^{e}, \mathbf{a}\right)(j \neq i)$. The semi-analytical description of turbo codes models the extrinsic MI $I\left(\tilde{\mathbf{a}}_{j}^{e}, \mathbf{a}\right)$ as the source of extra external information that has been passed through a hypothetical virtual channel, also often referred to as the extrinsic channel [7] directly to a decoder component's input. For each individual decoder, the LLRs $\tilde{\mathbf{a}}_{i}^{a}$ are constituted by the sum of all other decoders' extrinsic LLRs, each of which can be considered as though the original source information was repeated after passing through an independent extrinsic channel. Accordingly, all the extrinsic LLRs may be summed, which represents the decoding of a repetition code. Hence, the theory of information combining invoked for repetition codes [13] can be applied to analyze the MI combining in the context of our MCTCs. Based on [13, Chapter 5.3], the information combined in the presence of $(N-1)$ Additive White Gaussian Noise (AWGN) extrinsic channels ${ }^{2}$ may be expressed as

$$
\begin{aligned}
I_{c m b}^{A W G N}=\int_{j_{1}} \ldots \int_{j_{N-1}} p_{J_{1}}\left(j_{1}\right) \ldots p_{J_{N-1}}\left(j_{N-1}\right) & \\
& \cdot f_{N-1}^{\text {par }}\left(j_{1}, j_{2}, \ldots, j_{N-1}\right) d j_{1} \ldots d j_{N-1}
\end{aligned}
$$

where $p_{J_{1}}\left(j_{1}\right) \ldots p_{J_{N-1}}\left(j_{N-1}\right)$ is the joint probability density function (PDF) describing the MI distribution of the $(N-1)$ independent AWGN extrinsic channels and each $p_{J_{i}}\left(j_{i}\right)$ component represents the MI PDF of a specific extrinsic channel. Furthermore, $f_{N-1}^{p a r}\left(j_{1}, j_{2}, \ldots, j_{N-1}\right)$ is expressed in a closed form in [13], which represents the combined information, when the instantaneous MI values $j_{1}, j_{2}, \ldots, j_{N-1}$ gleaned from each extrinsic AWGN channel happen to emerge. The derivation of $p_{J}(j)$ for the AWGN extrinsic channel of turbo codes can be found in Appendix A.

By contrast, when $(N-1)$ individual extrinsic channels are modeled by Binary Erasure Channels (BECs), the combined a priori information exploited by our MCTCs having $N$ URC codes (equivalent to an $(N-1)$-component repetition code) may be formulated as [13]:

$$
I_{c m b}^{B E C}=1-\prod_{i=1}^{N-1}\left(1-I_{i}\right),
$$

where $I_{c m b}^{B E C}$ represents the post-combined information and $I_{i}$ denotes the $(N-1)$ information sources to be combined. Although Equation 5 was derived for the BEC, it may be applied with a reasonable accuracy for the AWGN extrinsic channel, as demonstrated below.

Using Equations 4 and 5, Figure 3 shows that there is only a slight difference between the MI obtained when combining two LLR sequences hypothetically received over the extrinsic BEC and over the extrinsic AWGN channel. More quantitatively, Figure 3 shows that the difference between the resultant MIs is small, which exceeds 0.01 only when the LLR sequences that are being combined have

\footnotetext{
${ }^{2}$ Such an AWGN extrinsic channel has the variance of $\sigma^{2}$ and a mean of $\pm \frac{\sigma^{2}}{2}[14]$.
}

MIs approaching 1 . However, since having near-unity MI implies that a low BER has already been achieved, we might reasonably conclude that for practical purposes, the extrinsic BEC model of MI combining offers a desirable low-complexity approximation of the Gaussian model.

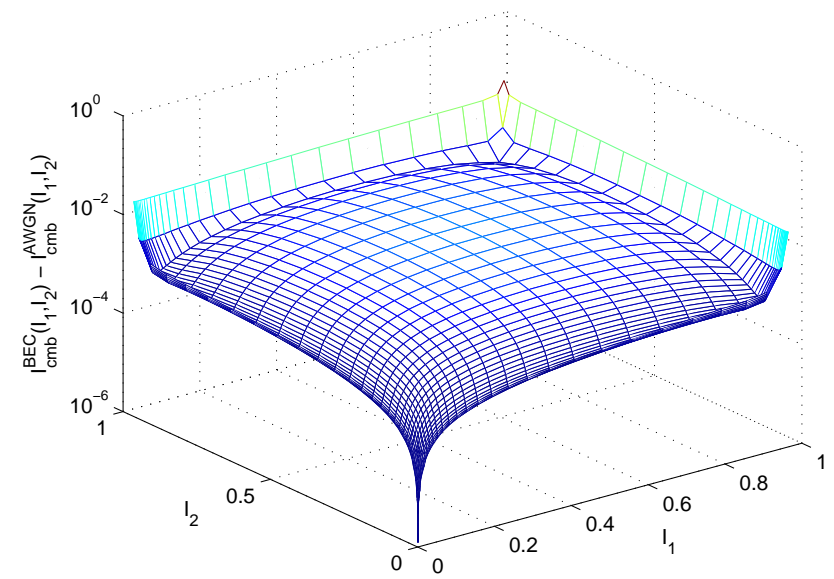

Fig. 3. The difference of the combined information, provided that the extrinsic channel of MCTCs is the BEC and the AWGN channel.

Hence, instead of Equation 4 we adopt the simpler Equation 5 as the expression of $I\left(\tilde{\mathbf{a}}_{i}^{a}, \mathbf{a}\right)$ in Equation 3. Let us consider $i=2$ as an example. The input a priori information $I\left(\tilde{\mathbf{a}}_{2}^{a}, \mathbf{a}\right)$ of Figure 2 is given by $I\left(\tilde{\mathbf{a}}_{2}^{a}, \mathbf{a}\right)=1-\left[1-I\left(\tilde{\mathbf{a}}_{1}^{e}, \mathbf{a}\right)\right]\left[1-I\left(\tilde{\mathbf{a}}_{3}^{e}, \mathbf{a}\right)\right] \cdots\left[1-I\left(\tilde{\mathbf{a}}_{N}^{e}, \mathbf{a}\right)\right]$. Using the iterative computation of the combined extrinsic information based on Equation 3 by taking into account the contributions of all the $(N-1)$ URC-BCJR decoders in the composite decoder of Figure 2, the composite EXIT function $I\left(\tilde{\mathbf{a}}_{1}^{a}, \mathbf{a}\right)=T_{\text {comp }}\left[I\left(\tilde{\mathbf{a}}_{1}^{e}, \mathbf{a}\right)\right]$ can be determined.

Figure 4 provides the resultant extended EXIT charts recorded for three different BPSK-modulated Rayleigh fading channel SNRs for MCTCs having rates of $R=\frac{1}{4}, \frac{1}{5}, \frac{1}{6}$ bits per channel use, when invoking component encoders employing only a single memory element. In order to view the increment of MI along with the number of components, the EXIT curves of MCTCs constructed from $N=2$ components are shown for these three SNRs. Note that in the EXIT charts of Figure 4, the dashed lines show the EXIT curves that were generated using the above-mentioned $10^{t h}$-order polynomial based EXIT-function models. These can be seen to be quite similar to the EXIT functions drawn in solid lines, which were obtained using the Gaussian LLR assumption and an interleaver length of 1,000, 000 bits. This demonstrates that our polynomial-fitting based EXIT chart modeling used for obtaining the composite EXIT function offers a desirable low-complexity alternative to the employment of the Gaussian LLR assumption.

\section{The SNR threshold of creating an open EXIT chart tunnel}

As mentioned in Section I, the creation of an open tunnel in an EXIT chart at a particular SNR is necessary for achieving iterative decoding convergence towards the maximum likelihood decoder's BER, provided that the interleaver is sufficiently long. We refer to the minimum SNR for which the EXIT chart has an open tunnel as 'the open tunnel SNR threshold'. Observe from Figures 4(a), (b) and (c) that 'the open tunnel SNR thresholds' of MCTCs using singledelay URC components and having throughputs of $R=\frac{1}{4}, \frac{1}{5}, \frac{1}{6}$ bits per channel use are $-5.05 \mathrm{~dB},-6.2 \mathrm{~dB}$ and $-7.05 \mathrm{~dB}$, respectively.

This motivates the investigations documented in Figure 5, which provides a scatter plot of the throughput or rate $R$ versus 'the open 
(a)

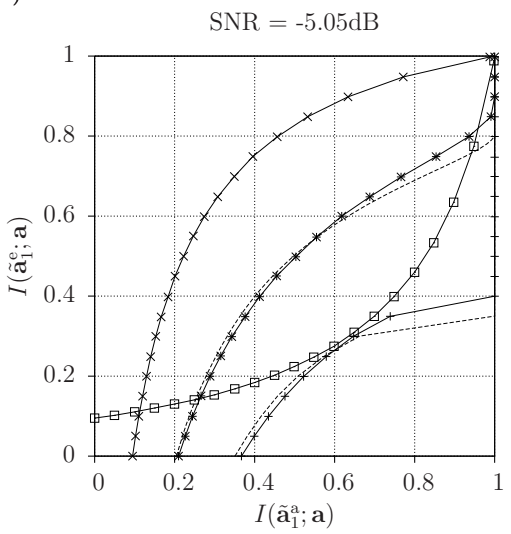

(b)

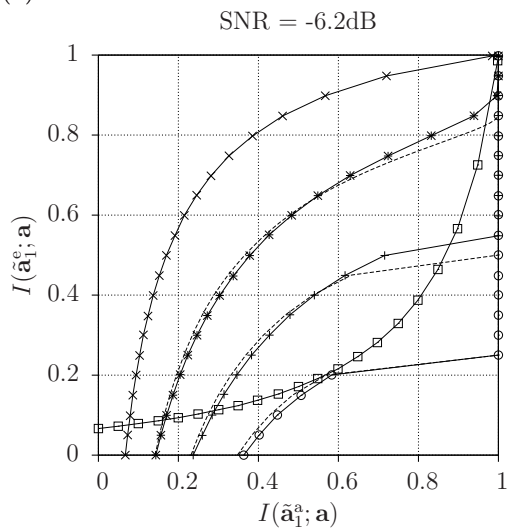

(c)

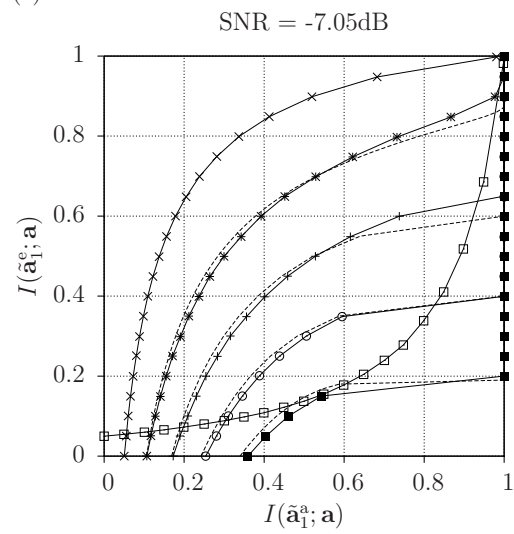

upper part $\square$ lower. $\mathrm{N}=2$ * lower, $\mathrm{N}=3$ * lower, $\mathrm{N}=4+$ lower, $\mathrm{N}=4+$ lower, $\mathrm{N}=6$.

Fig. 4. The extended EXIT charts for the MCTC structure of Figure 1 for $N=2,3,4,5,6$. The URCs have a memory-one generator and feedback polynomial of $(2,3)_{\text {Octal }}$. The communication channel is an uncorrelated Rayleigh channel. Then continuous line shows the EXIT-functions based on the Gaussian assumption, while the dashed line is its $10^{t h}$-order polynomial based approximation.

tunnel SNR' for MCTCs having a variety of generator and feedback polynomials. These polynomials were selected, because they were found to offer lower 'open tunnel SNR thresholds' than all other polynomials having the same length, at the specific throughputs considered. Figure 5 also plots the DCMC capacity of a BPSKmodulated Rayleigh fading channel as a function of its SNR [15]. It is the horizontal displacement of a particular symbol from this DCMC capacity plot that quantitatively characterizes the ability of the corresponding MCTC to approach the DCMC capacity. Note that upon using the $10^{t h}$-order polynomial based approximation of the EXIT function of the composite decoder, the resultant open tunnel SNR threshold becomes similar to that obtained using the Gaussian LLR approximation method associated with an iterleaver length of $1,000,000$ bits.

\section{Simulation Results}

In this section, we characterize the performance of the MCTCs designed and compare them to suitable TCTC benchmarkers. The encoders of these benchmarkers employ two URC encoders to generate the encoded bit sequences $b_{1}$ and $b_{2}$, which are transmitted the required number of times in order to achieve the desired throughput or rate of $R$ bits per channel use. For example, the output sequence is arranged to be $\left(b_{1}, b_{2}, b_{1}\right)$, when a throughput of $R=\frac{1}{3}$ bits per channel use is desired and $\left(b_{1}, b_{2}, b_{1}, b_{2}\right)$ for $R=\frac{1}{4}$. At the receiver, the LLRs corresponding to different replicas of the same encoded bit sequence are summed, before the iterative decoding process commences.

The 'open tunnel SNR thresholds' of MCTCs and TCTCs recorded when transmitting over BPSK-modulated Rayleigh fading channels may be compared in Figure 5. These results demonstrate that for throughputs below $R=\frac{1}{2}$ bits per channel use, MCTCs can create open EXIT chart tunnels at SNRs that are lower than those required for TCTCs. Furthermore, in order to create an open tunnel at these SNRs, the TCTCs are required to use the polynomial of $(8, F)_{o}$, which corresponds to $m=3$ memory elements in the URC encoder and $2^{m}=8$ states in the BCJR decoder. By contrast, MCTCs create open tunnels at the lowest SNRs, when using the polynomial $(4,7)_{o}$ at a throughput of $R=\frac{1}{3}$ and the polynomial $(2,3)_{o}$ at throughputs below $R=\frac{1}{3}$. These polynomials are associated with lower BJCR decoding complexities, since they correspond to as few as $2^{m}=4$ and 2 states, respectively.

While MCTCs benefit from having lower complexities per BCJR algorithm activation than TCTCs, they include more BCJR decoders. For this reason, Figure 6 compares the BERs that can be achieved

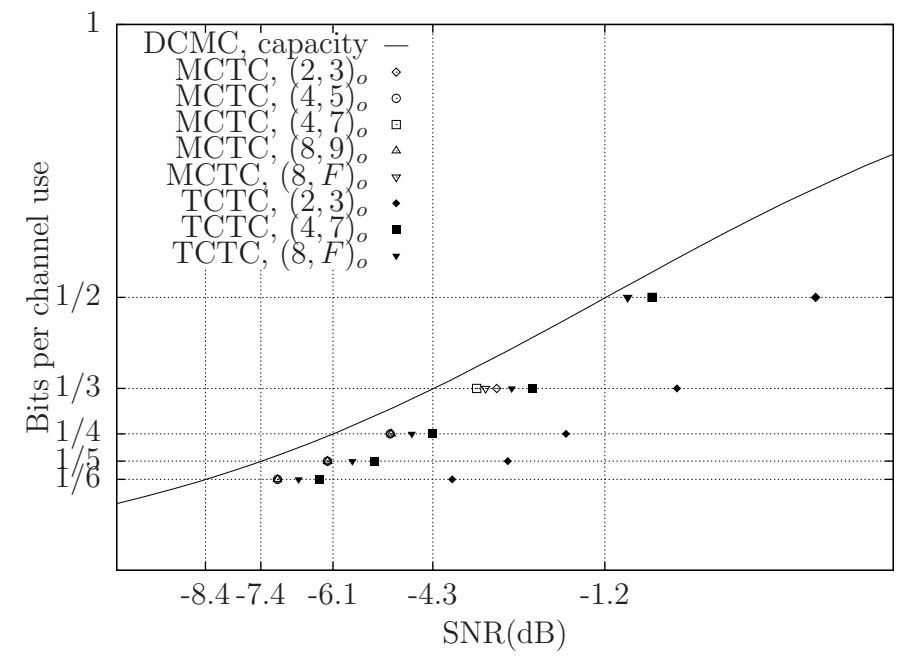

Fig. 5. Throughput versus open channel SNR threshold for MCTCs and TCTCs using various generator polynomials, when communicating over a BPSK-modulated Rayleigh fading channel.

by MCTCs and TCTCs, when fixed decoding complexities are used to recover information transmitted over a BPSK-modulated Rayleigh fading channel using an interleaver length of 2048 bits. Here, the complexity recorded in Figure 6 is defined as complexity $=2^{m} * N$, where $m$ is the number of memory elements in each URC encoder and $N$ is the total number of BCJR operations that are performed in the receiver, where a sequential decoder activation order is employed. As expected, Figure 6 shows that higher affordable complexities result in improved BER performances. The MCTCs have significantly steeper turbo cliffs and significantly lower error floors than the corresponding TCTCs at all the complexities considered. As a result, at a complexity of 48 , for example, the MCTCs offer $3.5 \mathrm{~dB}$ to $4.5 \mathrm{~dB}$ gain over the TCTCs at a BER of $10^{-6}$.

\section{CONCLUSIONS}

The area properties of the EXIT charts reveal that TCTCs inherently suffer from a capacity loss, when the overall turbo coding rate is required to be less than $\frac{1}{2}$. This motivates the design of MCTCs, which overcome this impediment by increasing the number of concatenated component codes, rather than reducing the component code rate below unity. In this paper, we provided a procedure for employing 2D EXIT charts for investigating $N$-component paralled concatenated codes, which would require $N$-dimensional EXIT charts. We demonstrated that 'the open tunnel SNR thresholds' of MCTCs are closer 
(a)

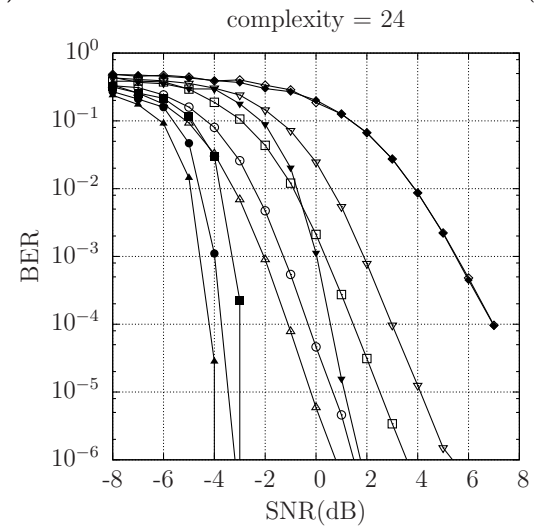

(b)

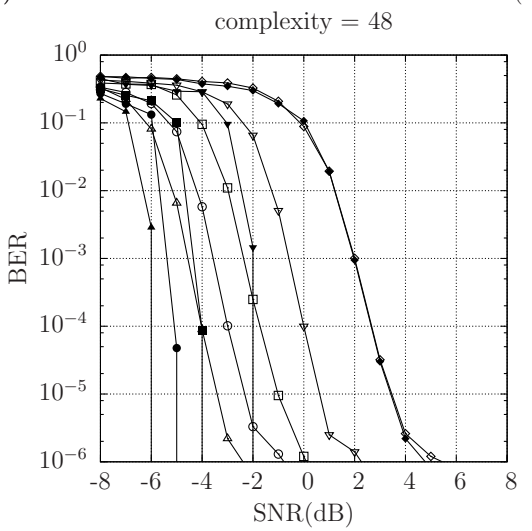

(c)

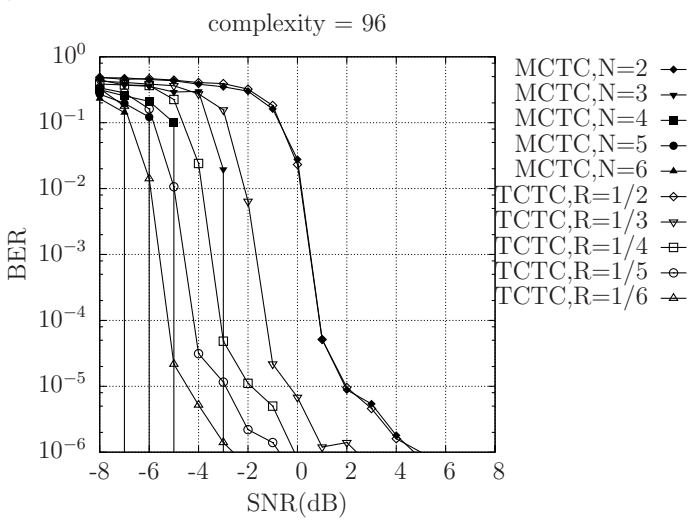

Fig. 6. The BER vs. SNR for MCTCs and TCTCs having different complexities. All the TCTCs having different rates $R$ employ the polynomial of $(8, F)_{o}$, while the $R=\frac{1}{3}$ MCTC employs $(4,7)_{o}$ and the MCTCs having $R<\frac{1}{3}$ employ $(2,3)_{o}$.

to the DCMC capacity compared to those of TCTCs. Furthermore, we showed that MCTCs offer significantly steeper BER turbo cliffs and lower error floors than TCTCs having the same coding rate and the same complexity.

\section{APPENDIX A}

In this appendix we highlight some theoretical foundations of information combining. It has been shown in [13] that any Binary Input Symmetric Memoryless Channel (BISMC) can be decomposed into a number of Binary Symmetric subChannels (BSCs) which are not further decomposable. The output of each BSC subchannel may have a different MI of $[1-h(\epsilon)]$, where $h(\epsilon)$ is the binary entropy function with respect to the BSC crossover probability $\epsilon$. Since an AWGN channel is a type of BISMC, it can be decomposed into an infinite number of BSC subchannels. The MI of the output of each BSC subchannel may be modeled by a random variable $J$ and the lower-case $j$ is used to denote its particular value. The Mutual Information Profile (MIP) is introduced to characterize the PDF of the MI of a BISMC, which is denoted by $p_{J}(j)$. Then, the MI at the output of the channel may be calculated using the expectation of $J$, expressed as $E(J)=\int_{j} j p_{J}(j) d j$.

If the extrinsic channel of the MCTCs is modeled as an AWGN channel having a variance of $\sigma^{2}$ and a mean of $\pm \frac{\sigma^{2}}{2}$ [14], the output of the channel, i.e. the extrinsic LLR $\tilde{\mathbf{a}}$, is equivalent to imposing independent Gaussian noise $\mathbf{n}$ on the transmitted source bits $\mathbf{a} \in\{0,1\}$. When decomposing this AWGN extrinsic channel into a series of continuous BSC subchannels, any pair of symmetric values $(+\tilde{a},-\tilde{a})$ may be considered to form a BSC subchannel. Hence, $(\tilde{a}>0)$ may be considered as the index of that BSC subchannel. The subchannel indicator $\tilde{A}$ is then a random variable with the value of $(\tilde{a}>0)$ about the subchannel index. The PDF of $\tilde{A}$ can be expressed by

$$
f_{\tilde{A}}(\tilde{a})=\frac{1}{\sqrt{2 \pi \sigma}}\left(e^{-\frac{\left(\tilde{a}+\sigma^{2} / 2\right)^{2}}{2 \sigma^{2}}}+e^{-\frac{\left(\tilde{a}-\sigma^{2} / 2\right)^{2}}{2 \sigma^{2}}}\right) \quad(\tilde{a}>0) .
$$

The corresponding mutual information of the subchannel $\tilde{a}$ is denoted as

$$
I(\tilde{a})=1-h\left(\frac{1}{1+e^{\tilde{a}}}\right),
$$

where $1 /\left(1+e^{\tilde{a}}\right)$ is the crossover probability of the subchannel $\tilde{a}$. Referring to [16], the MIP of the extrinsic AWGN channel may then be expressed as

$$
p_{J}(j)=\frac{d \tilde{a} \cdot f_{\tilde{A}}(\tilde{a})}{d I(\tilde{a})},
$$

where we have

$$
\tilde{a}=\ln \left(\frac{1}{h^{-1}(1-j)}-1\right) ; \quad \frac{d I(\tilde{a})}{d \tilde{a}}=\frac{e^{\tilde{a}} \cdot \log _{2}\left(e^{\tilde{a}}\right)}{\left(1+e^{\tilde{a}}\right)^{2}} .
$$

\section{REFERENCES}

[1] D. Divsalar and F. Pollara, "Multiple turbo codes," in IEEE Military Communications Conference MILCOM '95, Conference Record, vol. 1, 5-8 Nov. 1995, pp. 279-285.

[2] D. Divsalar, "Multiple turbo codes for deep-space communications," Jet Propulsion Lab., Pasadena, CA, Tech. Rep., 1995.

[3] E. Boutillon and D. Gnaedig, "Maximum spread of D dimensional multiple turbo codes," IEEE Transactions on Communications, vol. 53, no. 8, pp. 1237-1242, Aug. 2005.

[4] A. Huebner, K. S. Zigangirov, and D. J. Costello, "A new cycle-based joint permutor design for multiple turbo codes," IEEE Transactions on Communications, vol. 54, no. 6, pp. 961-965, June 2006.

[5] H. Chen, R. Maunder, and L. Hanzo, "Multi-level turbo decoding assisted soft combining aided hybrid ARQ," Vehicular Technology Conference Spring (VTC 2010-Spring), 2010 IEEE 71st, pp. 1-5, 16-19 May 2010.

[6] S. X. Ng, Y. Li, and L. Hanzo, "Distributed turbo trellis coded modulation for cooperative communications," in Proceeding of IEEE International Conference on Communications ICC '09, 14-18 June 2009, pp. 1-5.

[7] A. Ashikhmin, G. Kramer, and S. ten Brink, "Extrinsic information transfer functions: model and erasure channel properties," IEEE Transactions on Information Theory, vol. 50, no. 11, pp. 2657-2673, Nov. 2004.

[8] L. Hanzo, T. H. Liew, and B. Yeap, Turbo Coding, Turbo Equalisation and Space-Time Coding for Transmission over Fading Channels. John Wiley \& Sons, Ltd, 2002.

[9] D. Divsalar, S. Dolinar, and F. Pollara, "Serial concatenated trellis coded modulation with rate-1 inner code," in Proceeding of IEEE Global Telecommunications Conference GLOBECOM '00, vol. 2, 27 Nov.-1 Dec. 2000, pp. 777-782.

[10] S. Huettinger and J. Huber, "Analysis and design of power-efficient coding schemes with parallel concatenated convolutional codes," IEEE Transactions on Communications, vol. 54, no. 7, pp. 1251-1258, July 2006.

[11] S. ten Brink, "Convergence behavior of iteratively decoded parallel concatenated codes," IEEE Transactions on Communications, vol. 49, no. 10, pp. 1727-1737, Oct. 2001.

[12] L. Hanzo, O. Alamri, M. El-Hajjar, and N. Wu, Near-Capacity MultiFunctional MIMO Systems. John Wiley \& Sons, Ltd, 2009.

[13] I. Land and J. Huber, Information Combining. Now Publishers Inc., 2006.

[14] J. Hagenauer, "The EXIT chart - introduction to extrinsic information transfer in iterative processing," in Proceeding of 12th European Signal Processing Conference (EUSIPCO, 2004, pp. 1541-1548.

[15] L. Hanzo, S. X. Ng, T. Keller, and W. Webb, Quadrature Amplitude Modulation. John Wiley \& Sons, Ltd, 2004.

[16] J. Huber, T. Hehn, I. Land, and P. A. Hoeher, "Mutual information profile of a BISMC with applications," Preceeding of Conference on Information Science and Systems, Maryland USA, pp. 95-102, 2005. 